\title{
EVALUACIÓN DE ESTRÉS EN EL PECARI TAJACU SOMETIDO A DOS DENSIDADES DE POBLACIÓN
}

\author{
STRESS EVALUATION IN PECARI TAJACU UNDER TWO STOCKING DENSITIES \\ Montes-Pérez, R.C. ${ }^{1 *}$, A.L. Solís-Sosa ${ }^{1}$, J. Yokoyama-Kano ${ }^{1}$, J.M. Mukul-Yerves ${ }^{1}$ \\ y J.C. Segura-Correa ${ }^{1}$
}

${ }^{1}$ Facultad de Medicina Veterinaria y Zootecnia. Universidad Autónoma de Yucatán. Apartado Postal 4-116. CP 97100. Mérida. Yucatán. México. *mperez@tunku.uady.mx

\section{Palabras ClaVe ADICIONALES}

Cortisol. Conductas agonísticas. Espacio vital. Pecarí de collar.

\section{RESUMEN}

El objetivo de este trabajo fue evaluar el nivel de estrés producido por dos densidades de población en pecaríes de collar (Pecari tajacu) en cautiverio. Se formaron cuatro grupos de animales $(N=20)$, todos adultos; un grupo de hembras $(n=5)$ y otro de machos $(n=5)$ se colocaron en corrales con una densidad de población de $20 \mathrm{~m}^{2} /$ animal, y los otros dos grupos en densidades de $10 \mathrm{~m}^{2} /$ animal durante 30 días. Se tomaron muestras sanguíneas a cada animal a los 0,15 y 30 días y se registraron las cantidades y tipo de lesiones corporales por animal a los 3, 15 y 30 días del periodo de estudio. Los niveles de cortisol sanguíneo se midieron a través de pruebas de radioinmunoanálisis. Los datos se analizaron para cada día de muestreo utilizando pruebas de Fisher o análisis de varianza. No se encontraron efectos de sexo y densidad en el número de animales lesionados. El análisis de varianza no mostró efecto de densidad $(p>0,05)$ sobre la cantidad de lesiones o niveles de cortisol, en ninguno de los días evaluados. Sin embargo, la interacción sexo $x$ densidad tuvo efecto sobre cortisol los días 15 $(p<0,03)$ y $30(p<0,0005)$. En promedio, las hembras tuvieron mayores niveles de cortisol que los machos (10,1 y 7,5 mg/dl). La interacción se debió a una mayor concentración de cortisol en las hembras a la densidad de $10 \mathrm{~m}^{2} / \mathrm{animal}$ en comparación con los machos; pero no hubo diferen-

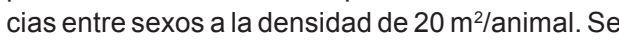
concluye que los efectos del estrés, medido mediante los niveles de cortisol en sangre, dependen del sexo y densidad poblacional.

\section{Additional KeYWORDS}

Cortisol. Agonistic behavior. Stress. Vital area. Collared peccary.

\section{SUMMARY}

The objective of this study was to evaluate the level of stress produced by two stocking densities in the collared peccary (Pecari tajacu) in captivity. Four groups of adult animals $(N=20)$ were established: one group of females $(n=5)$ and other of males $(n=5)$ were correlated under a stocking density of $20 \mathrm{~m}^{2} / \mathrm{animal}$, and the other two groups under a density of $10 \mathrm{~m}^{2} /$ animal, during 30 days. Three blood samples per animal were taken at 0 , 15 and 30 days and the amount and type of body lesions were registered at 3,15 and 30 days of the study period. Cortisol levels were measured by radioimmunoanalysis tests. Data were analyzed for each sampling day using Fisher or analysis of variance tests. There were not effects of sex and stocking density on the number of animals with lesions. The analysis of variance showed no effect of density $(p>0.05)$ on the amount of lesions or cortisol level at any of the evaluation days. However the interaction of sex $x$ density was significant on cortisol at days $15(p<0.03)$ and 30 $(p<0.0005)$. In average females had higher levels of cortisol than males (10.1 and $7.5 \mathrm{mg} / \mathrm{dl})$. The interaction was due to a greater concentration of cortisol in females kept under a density of $10 \mathrm{~m}^{2} /$ animal in comparison with males; but there was no differences between sexes at the stocking density of $20 \mathrm{~m}^{2} /$ animal. It is concluded that the effect of stress, measured through the levels of cortisol in blood, depends of sex and stocking density.

\section{INTRODUCCIÓN}

Uno de los aspectos fundamentales para 
el mantenimiento de los animales domésticos y animales silvestres en cautiverio, es la superficie de alojamiento. Existe un reporte de Houston (2006) que indica que la superficie vital recomendada para cerdos y pecaríes de collar (Pecari tajacu) es de 20 a 60 pies cuadrados por animal; sin embargo, no existen datos publicados sobre experimentos en pecaríes de collar dirigidos a considerar los aspectos conductuales y el probable estrés de los animales por las dimensiones del espacio vital. El objetivo del presente trabajo fue comparar el nivel de estrés a través del tipo y cantidad de lesiones corporales y, los niveles de cortisol sanguíneo en machos y hembras sometidos a densidades de 20 y $10 \mathrm{~m}^{2} /$ animal.

\section{MATERIAL Y MÉTODOS}

El presente trabajo se realizó en la Unidad de Manejo Animal de la Universidad Autónoma de Yucatán, ubicada al sur de Mérida, Yucatán, México. La región geográfica presenta clima tropical subhúmedo Awo( $\left.i^{\prime}\right) g$ con lluvias en los meses de mayo a octubre (Duch, 1988). Los pecaríes utilizados en este estudio nacieron y se criaron en cautiverio y fueron descolmillados.

Se formaron cuatro grupos de animales adultos: el grupo 1 constituido por 5 hembras con peso vivo de 17,3 $\pm 1,3 \mathrm{~kg}$ (media \pm desviación estándar); las cuales estuvieron confinadas a una densidad de $10 \mathrm{~m}^{2} /$ animal; el grupo 2 por 5 hembras de 21, $0 \pm 1,6$ $\mathrm{kg}$ de peso, a una densidad de $20 \mathrm{~m}^{2}$ /animal; ambos grupos de hembras presentaron eda- des entre 1 a 5 años, las cuales se encontraban en edad reproductiva (Sowls, 1966a). El grupo 3, por 5 machos adultos con peso de $20,4 \pm 2,3 \mathrm{~kg}$; a una densidad de $10 \mathrm{~m}^{2} /$ animal y el grupo 4 por 5 machos de 18,2 $\pm 1,5 \mathrm{~kg}$, a una densidad de $20 \mathrm{~m}^{2} /$ animal; cuyas edades oscilaron entre 1,5 y 5 años de edad, éstos también se encontraban en edad reproductiva (Ojasti, 1993). El experimento duró 30 días.

Los pecaríes fueron inmovilizados por contención química, utilizando una inyección intramuscular de clorhidrato de ketamina a dosis de $20 \mathrm{mg} / \mathrm{kg}$. El nivel de estrés se midió mediante el registro de lesiones y concentraciones de cortisol sanguíneo. Se tomaron muestras sanguíneas de cada animal el día 0,15 y 30 de iniciado el experimento y se registraron el número y tipo de lesiones corporales en los animales a los 3, 15 y 30 días de iniciada la prueba.

El tipo y cantidad de lesiones en el cuerpo se registraron de acuerdo con el método de Ekesbo citado por Fraser y Broom (1990). Las lesiones se registraron en cuatro categorías, tipo I: golpes y raspones en la piel de los pecaríes; tipo II: golpes y raspones pero con inflamación; tipo III: traumatismos inflamados y heridas con sangre y IV: lesiones graves ocasionadas por mutilaciones o fracturas. Se colectaron muestras de sangre de la vena safena, que se depositaron en tubos con anticoagulante, para separar el plasma por centrifugación. Los niveles de cortisol se midieron por radioinmunoanálisis en fase sólida con un estuche comercial.

El número de animales lesionados se

Tabla I. Número de pecaries de collar lesionados por densidad, sexo y día de muestreo. (Number of collared peccaries hurt by density, sex and day of sample).

\begin{tabular}{lllllllr}
\hline \multirow{2}{*}{ Densidad } & \multicolumn{2}{c}{ Día 3 } & \multicolumn{2}{c}{ Día 15 } & \multicolumn{2}{c}{ Día 30 } \\
& Sexo & Si & No & Si & No & & Si \\
\hline \multirow{2}{*}{$20 \mathrm{~m}^{2} /$ manimal } & Macho & 4 & 1 & 4 & 1 & 5 & 0 \\
& Hembra & 1 & 4 & 0 & 5 & 3 & 2 \\
& Macho & 1 & 4 & 2 & 3 & 1 & 4 \\
& Hembra & 3 & 2 & 3 & 2 & 3 & 2 \\
\hline
\end{tabular}

Archivos de zootecnia vol. 58, núm. 223, p. 464. 
ESTRÉS EN EL PECARI TAJACU SOMETIDO A DOS DENSIDADES DE POBLACIÓN

Tabla II. Número y tipo de lesión por densidad, sexo y por día de medición, en pecaríes de collar. (Number of lesions by density, sex and type of damage by day of trial in collared peccaries).

\begin{tabular}{|c|c|c|c|c|c|c|c|}
\hline \multirow[b]{2}{*}{ Densidad } & \multirow[b]{2}{*}{ Sexo } & \multicolumn{2}{|c|}{ Día 3} & \multicolumn{2}{|c|}{ Día 15} & \multicolumn{2}{|c|}{ Día 30} \\
\hline & & $1^{*}$ & $\|^{*}$ & $1^{*}$ & $\|^{*}$ & $I^{*}$ & $\|^{*}$ \\
\hline \multirow[t]{2}{*}{$10 \mathrm{~m}^{2} /$ animal } & Macho & 8 & 3 & 7 & 1 & 7 & 0 \\
\hline & Hembra & 1 & 0 & 0 & 0 & 4 & 1 \\
\hline \multirow[t]{2}{*}{$20 \mathrm{~m}^{2} /$ animal } & Macho & 1 & 0 & 3 & 0 & 1 & 0 \\
\hline & Hembra & 1 & 1 & 4 & 3 & 3 & 0 \\
\hline
\end{tabular}

*Tipos de lesión: I= golpes y raspones; II= golpes y raspones con inflamación marcada.

analizó mediante la prueba exacta de Fisher y la cantidad de lesiones por animal y los niveles de cortisol a través de análisis de varianza en un experimento factorial, probando como factores sexo y densidad poblacional. Los análisis estadísticos se realizaron por separado para los tres días de evaluación.

\section{RESULTADOSYDISCUSIÓN}

El número de pecaríes lesionados y cantidad de lesiones por día de muestreo, se presentan en las tablas I y II. No se encontró efecto significativo $(\mathrm{p}>0,05)$ por la densidad o sexo sobre el número de animales lesionados ni la cantidad de lesiones. Los niveles de cortisol en plasma sanguíneo de los animales por densidad, sexo y día de muestreo se presentan en la tabla III.

Se observa una tendencia mayor de lesiones en los machos a densidad de $10 \mathrm{~m}^{2} /$ animal respecto a la hembras, mostrando éstas mayor cantidad de lesiones a densidad de $20 \mathrm{~m}^{2}$ /animal; situación que en los machos podría ser atribuido a la conducta territorial que éstos tienen, debido a que permanecen aislados del grupo matriarcal y consecuentemente al reducirse el espacio vital, tienden a pelear por su territorio (Sowls, 1996b); sin embargo, en ningún caso se encontró lesión de tipo III y IV, por lo que las consecuencias de las conductas agonísticas que se presentaron fueron leves.

Por razones de bienestar animal, sería recomendable mantener a los pecaríes en densidades de $20 \mathrm{~m}^{2} /$ animal, puesto que a esta densidad se producen menos actividades agonísticas. En las cerdas salvajes, éstas tienden a conservar una conducta matriarcal (British Wild Boar, 2006), y por tanto la hembra dominante obliga a las demás a mantenerse en grupo conforme aumenta la superficie disponible en corral.

Los niveles de cortisol sanguíneo que

Tabla III. Medias y desviaciones estándares por densidad, sexo y día de medición para niveles de cortisol en sangre de pecaries de collar. (Means and standard deviations by density, sex and day of trial for blood-cortisol levels of collared peccaries).

\begin{tabular}{|c|c|c|c|c|c|}
\hline \multirow[b]{2}{*}{ Densidad } & \multirow[b]{2}{*}{ Sexo } & \multicolumn{4}{|c|}{ Cortisol ( $\mu \mathrm{g} / \mathrm{dl})$} \\
\hline & & Día 0 & Día 15 & Día 30 & Promedio \\
\hline \multirow[t]{2}{*}{$10 \mathrm{~m}^{2} /$ animal } & Macho & $5,38 \pm 2,61$ & $7,14 \pm 1,17$ & $6,67 \pm 2,05$ & $6,40 \pm 2,04$ \\
\hline & Hembra & $10,87 \pm 3,32$ & $12,56 \pm 2,71$ & $12,09 \pm 2,07$ & $11,84 \pm 2,07$ \\
\hline \multirow[t]{2}{*}{$20 \mathrm{~m}^{2} /$ animal } & Macho & $7,82 \pm 3,03$ & $8,13 \pm 1,48$ & $9,73 \pm 2,11$ & $8,56 \pm 2,10$ \\
\hline & Hembra & $8,73 \pm 4,78$ & $8,72 \pm 3,17$ & $7,76 \pm 1,21$ & $8,40 \pm 1,21$ \\
\hline
\end{tabular}




\section{MONTES-PÉREZETAL.}

presentaron los machos y hembras fueron mayores a lo informado por Hughes y Lytle (1975), que fueron de $6,67 \mu \mathrm{g} / 100 \mathrm{ml}$ para hembras, y de 7,5 $\mu \mathrm{g} / 100 \mathrm{ml}$ para machos adultos. Sin embargo, la comparación de niveles de cortisol de este trabajo con los de Hughes y Lytle (1975) no puede ser efectuada, debido a que estos autores midieron cortisol con método colorimétrico, por lo tanto los niveles de cortisol reportados aquí,

\section{BIBLIOGRAFÍA}

British Wild Boar. 2006. Breeding wild boar. www.britishwildboar.org.uk/breeding.htm Acceso: 23 de mayo de 2006.

Duch, G.J. 1988. La conformación territorial del estado de Yucatán. Universidad Autónoma de Chapingo. Centro Regional de la Península de Yucatán. Mérida, Yucatán. México. p. 188-195.

Fraser, A.F. and D.M. Broom. 1990. Animal Behaviour and Welfare. Baillière Tindall. London. UK. 322 p.

Houston, W. 2006. Estándares de zoológicos para el mantenimiento de cerdos salvajes en cautividad. http://zcog.org/zcog\%20frames/library captivity files/Cerdos $\% 20$ Salvajes/Cerdos $\%$ son comparables cuando se utiliza radioinmunoanálisis. Aunque los niveles de cortisol fueron diferentes entre sexos, la presencia de un efecto significativo en la interacción sexo $\mathrm{x}$ densidad sobre niveles de cortisol a los días $15(\mathrm{p}<0,03)$ y $30(\mathrm{p}<0,0005)$, indica que los efectos del estrés, medido mediante los niveles de cortisol en sangre, dependen del sexo en combinación con la densidad poblacional.

20Salvajes.htm Acceso: 22 de mayo de 2006 Hughes, J.P. and I.M Lytle. 1975. Corticosteroids of the peccary (Dicotyles tajacu). Gen. Comp. Endocr., 26: 277-280.

Ojasti J. 1993. Utilización de la fauna silvestre en América. Guía n² 25. FAO. Roma. p. 100-103.

Sowls, L.K. 1966a. Reproduction in the collared peccary (Tayassu tajacu). In: J.W. Rowland (eds). Comparative biology of reproduction in mammals. Zool. Soc. London. p. 155-172.

Sowls, L.K. 1996b. Javelinas and other peccaries: Their biology, management, and use. Second ed. Texas A\&M University Press. Tucson, Arizona. p. 145. 